Matthias Berg

\title{
Singularität versus Serialität? Überlegungen zu Münchner Universitätsreden anlässlich von Hochschuljubiläen im 19. Jahrhundert
}

\begin{abstract}
This article aims to compare speeches that were delivered in the $19^{\text {th }}$ century at the Ludwig Maximilian University and the Technical University of Munich on an annual and routine base with speeches for anniversaries and other exceptional occasions. In so doing, this contribution sheds light on the role the academic speech culture played in endowing university culture with meaning, a typical feature of university anniversaries and other festivities. So far, the research perspective on university speeches has hardly been explicitly linked to the increased interest of university historiography in academic festivities. The article first addresses the seemingly obvious difference between the serial nature of annual university (for example, at the inauguration of the rector) and the singularity of speeches on the occasion of an anniversary. In a second step, the author analyses whether the speakers of jubilee speeches had really designed them for a singular and exception purpose and whether they succeeded in escaping the attraction that the seriality of the annual speeches and their parameters emanated.
\end{abstract}

\section{Einleitung}

Mit dem verstärkten Interesse an akademischen Festkulturen wie auch mit der Erforschung von Universitätsreden ist die jüngere Universitätsgeschichtsschreibung zwei verwandten Forschungsperspektiven gefolgt, ohne diese - soweit ich sehe - bislang explizit aufeinander zu beziehen. ${ }^{1}$ Der entsprechende Anlass einer Rede im universitären Rahmen fand zwar durchaus Erwähnung. Ebenso berücksichtigten Studien zur Festkultur selbstverständlich die diesbezüglichen Ansprachen. Der Anteil akademischer Redekultur an der in Hochschuljubiläen sich widerspiegelnden universitären Sinnstiftung jedoch wurde eher gestreift,

1 Vgl. z.B. Becker, Thomas P.: Jubiläen als Orte universitärer Selbstdarstellung. Entwicklungslinien des Universitätsjubiläums von der Reformationszeit bis zur Weimarer Republik. In: Universität im öffentlichen Raum. Hrsg. von Rainer Christoph Schwinges. Basel 2008 (Veröffentlichungen der Gesellschaft für Universitäts- und Wissenschaftsgeschichte 10). S. 77-107, hier S. 87.

Ә OpenAccess. (C) 2022 Matthias Berg, publiziert von De Gruyter. (cc))BY Dieses Werk ist lizenziert unter einer Creative Commons Namensnennung 4.0 International Lizenz. https://doi.org/10.1515/9783110731378-013 
zumindest nicht gesondert hervorgehoben. ${ }^{2}$ Es fällt rasch ins Auge, dass die noch junge, aber beständig zunehmende Forschung zur Geschichte von Hochschuljubiläen sich vielfältigen, gern auch bislang weniger beachteten Aspekten widmet. ${ }^{3}$ Tatsächlich waren (und sind) ein öffentliches Aufsehen erregender Festumzug oder eine aufwendig hergestellte Festschrift besondere Ereignisse, während die akademische Rede offenbar und durchaus zu Recht dem universitären Alltag zugeschlagen wird. Hier möchte der Beitrag ansetzen und nach dem Bedingungsbeziehungsweise Spannungsverhältnis beider Phänomene fragen, mithin Alltag und Ausnahme universitären Redens in ein Verhältnis miteinander setzen.

Eine derartige Inspektion kann, selbst wenn sie auf Münchner Universitätsreden beschränkt bleibt, allenfalls punktuell erfolgen. ${ }^{4}$ Ermöglicht wird ein solches, selektives Vorgehen durch den in einem Projekt des Münchner Universitätsarchivs in unvergleichbar dichter Weise erschlossenen und digital verfügbaren Bestand aller - soweit überliefert - zwischen 1800 und 1968 an der Ludwig-Maximilians-Universität gehaltenen Rektorats- und Universitätsreden. ${ }^{5}$ Für den in den Blick genommenen Zeitraum handelt es sich, fasst man das 19. Jahrhundert streng kalendarisch als bis zum Jahr 1899 reichend auf, um etwa achtzig Reden zum Rektoratsantritt, um vierzig anlässlich des Stiftungsfestes gehaltene Ansprachen und weitere knapp fünfzig zu verschiedenen Anlässen vorgetragene „Gelegenheitsreden“. Die zwischen 1830 und 1867 erstatteten Jahresberichte enthalten ebenfalls Reden, zudem werden zugunsten eines institutionellen Vergleichs auch an der seit 1877 unter diesem Namen firmierenden Technischen

2 Vgl. Kreis, Georg: Tradition, Variation und Innovation. Die Basler Universitätsjubiläen im Lauf der Zeit, 1660 -1960. In: Schweizerische Zeitschrift für Geschichte 60 (2010). S. 437-474.

3 Neben der die Feierlichkeiten begleitenden Musik beziehungsweise den Chören von Universitäten auch der studentischen Fastnacht oder der „Rolle des Talars“, vgl. entsprechend Beiträge in: Kintzinger, Martin, Wolfgang Eric Wagner u. Marian Füssel. (Hrsg.): Akademische Festkulturen vom Mittelalter bis zur Gegenwart. Zwischen Inaugurationsfeier und Fachschaftsparty. Basel 2019 (Veröffentlichungen der Gesellschaft für Universitäts- und Wissenschaftsgeschichte 15); ein gesondert den Reden gewidmeter Beitrag findet sich in dem Band hingegen nicht, ebenso vgl. Dhondt, Pieter (Hrsg.): University Jubilees and University History Writing. A Challenging Relationship. Leiden 2015 (Scientific and Learned Cultures and their Institutions 13).

4 Ebenfalls ausgespart bleiben methodische Überlegungen zu den Besonderheiten der Quellengattung Rede, vgl. den eingehenden Problemaufriss von Baumgärtner, Ulrich: Reden als historische Quellen. Anmerkungen zu neueren Publikationen zur politischen Rede und zum historiographischen Umgang mit rhetorischen Texten. In: Historisches Jahrbuch 122 (2002). S. 559 596.

5 Vgl. den Bestand unter https://www.universitaetsarchiv.uni-muenchen.de/digitalesarchiv/in dex.html (16.12.2020); sowie erläuternd Stein, Claudius: Onlinepräsentation des LMU-RedenKorpus. In: Der rhetorische Auftritt. Redekultur an der Ludwig-Maximilians-Universität München. Rektorats- und Universitätsreden 1826-1968. Hrsg. von ders. München 2016. S. 37-45. 
Hochschule München gehaltene Ansprachen berücksichtigt. Alles in allem ergibt dies ein Korpus von deutlich über zweihundert Reden - mit entsprechendem Zuwachs, je nachdem ob man den historisch gedeuteten Übergang zum 20. Jahrhundert in den Jahren 1914, 1917 oder 1918 anzusetzen beliebt.

\section{Universitäten und ihre Reden}

Geredet wurde (und wird) an Universitäten selbstverständlich in Permanenz; die „Rede“ als gesonderte, feierliche Veranstaltung, vor der universitären oder weiteren Öffentlichkeit, zählte (und zählt) zu den festen Bestandteilen des universitären Lebens. Mehr noch, Reden strukturieren den Alltag der Universität als Institution. Im deutschsprachigen Raum begannen die Universitäten zum Beginn des hier betrachteten Zeitraum des 19. Jahrhunderts, „einmal im Jahr mit einer Rede ihres Rektors vor die Öffentlichkeit zu treten, ein feierlicher Akt, der sich

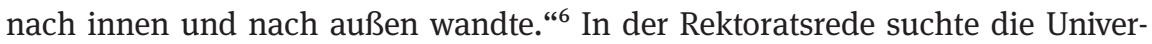
sität, so hat es Dieter Langewiesche - auf dessen Forschungen zu den Rektoratsreden in diesem Zusammenhang an allererster Stelle hinzuweisen ist $^{7}$ pointiert zusammengefasst, ihren Standort in der Wissenschaft und in der Gesellschaft zu bestimmen sowie dieses Selbstbild der Öffentlichkeit zu vermitteln. Es kann dabei, wie es Langewiesche für die Rektoratsreden typisierend vorgenommen hat, zwischen Gesellschafts- und Fachreden unterschieden werden. Während sich letztere um Wissensvermittlung bemühten, thematisch vor allem Forschungsfragen verhandelten und dem klassischen wissenschaftlichen Vortrag eng verwandt waren, verfolgte die Gesellschaftsrede andere Zwecke: Ihr Gegenstand waren in der Regel Fragen des Verhältnisses von Wissenschaft be-

6 Langewiesche, Dieter: Zum Selbstbild der Universität. Leipziger Rektoratsreden im Kaiserreich und in der Weimarer Republik. In: Reden zur feierlichen Übergabe der Leipziger Rektoratsreden 1871-1933. Hrsg. von Universität Leipzig. Leipzig 2009 (Leipziger Universitätsreden Neue Folge 108). S. $15-26$, hier S. 15.

7 Vgl. Langewiesche, Dieter: Selbstbilder der deutschen Universität in Rektoratsreden. Jena spätes 19. Jahrhundert bis 1948. In: Jena. Ein nationaler Erinnerungsort? Hrsg. von Jürgen John u. Justus H. Ulbricht. Köln 2007. S. 219-243; ders.: Die ,Humboldtsche Universität‘ als nationaler Mythos. Zum Selbstbild der deutschen Universitäten in ihren Rektoratsreden im Kaiserreich und in der Weimarer Republik. In: Historische Zeitschrift 290 (2010). S. 53-91; ders.: Humboldt als Leitbild? Die deutsche Universität in den Berliner Rektoratsreden seit dem 19. Jahrhundert. In: Jahrbuch für Universitätsgeschichte 14 (2011). S. 15-37; ders.: Die Rektoratsreden an den Universitäten im deutschen Sprachraum. In: Der rhetorische Auftritt. Redekultur an der LudwigMaximilians-Universität München. Rektorats- und Universitätsreden 1826-1968. Hrsg. von Claudius Stein. München 2016. S. 21-36. 
ziehungsweise wissenschaftlichen Institutionen zu Gesellschaft und Politik; nicht selten in instrumenteller und durchaus eigennütziger Absicht. Nationale Identitätsstiftung rückte seit der Mitte des 19. Jahrhunderts hier vielfach in den Mittelpunkt, zumal wenn sich politische, nicht selten auch militärische Ereignisse vor das universitäre Bild schoben. Vollkommen frei von solchen außeruniversitären Bezügen blieben auch die einer wissenschaftlichen Fragestellung gewidmeten Fachreden selbstredend nicht.

All dies trifft im Kern auch auf sämtliche anderen an Hochschulen jedweder Art gehaltenen Reden zu - ob zu Stiftungsfesten, anlässlich von Reichsgründungsfeiern oder eben auch zu Jubiläen, deren Anlass nicht allein, aber oftmals die Geschichte der Institution stiftete. Man wird vom Beginn des 19. Jahrhunderts bis in die heutige Zeit kaum ein Jubiläum ohne solcherart offenkundig unverzichtbare Ansprachen finden können. Was also unterscheidet die Rede anlässlich eines hochschulgeschichtlichen Jubiläums von den anderen benannten Ansprachen, was gibt ihr eine möglicherweise besondere Kontur? Unmittelbar ins Auge fällt der Unterschied zwischen der jährlichen Wiederholung, der Serialität der Rektoratsreden, ebenso jener Ansprachen zu den ebenfalls vielfach jährlich begangenen Stiftungsfesten, und der Singularität der Reden anlässlich eines Hochschuljubiläums. Diese wurden (und werden) zumeist in Schritten von 25, fünfzig oder hundert Jahren begangen und nahmen (und nehmen) Zeiträume von oftmals mehreren Jahrhunderten in den Blick. Allerdings bedarf dieser zunächst unmittelbar einleuchtende Unterschied auch einer tatsächlichen Überprüfung: Gestalteten die Redner der Jubiläumsansprachen diese auch wirklich singulär, vermochte sich diese Form von Universitätsreden von der Sogwirkung der Serialität ihrer Artgenossen zu emanzipieren? Denn Serialität konnte entweder erwünschte Orientierung oder routinierte Langeweile bedeuten. Schließlich ergab es sich nicht immer, dass die dem etablierten Universitätskalender folgenden Rektoratsreden miteinander in Beziehung traten, unmittelbar aufeinander zu antworten schienen wie in den Jahren 1870 und 1871, als an der Münchner LudwigMaximilians-Universität zuerst der Historiker Wilhelm Giesebrecht über „den Einfluß der deutschen Hochschulen auf die nationale Entwickelung“ sprach, und der Theologe Ignaz von Döllinger im Folgejahr zum Antritt seines Rektorats in gleichsam umgekehrter Richtung die „Bedeutung der großen Zeitereignisse für die deutschen Hochschulen“ erörtern konnte. ${ }^{8}$

8 Giesebrecht, Wilhelm: Ueber den Einfluß der deutschen Hochschulen auf die nationale Entwickelung. München 1870; Döllinger, Ignaz von: Die Bedeutung der großen Zeitereignisse für die deutschen Hochschulen. In: ders.: Akademische Vorträge. Bd. 3. München 1891. S. 11-38; zu Giesebrecht an der Münchner Universität vgl. Schieffer, Rudolf: Wilhelm von Giesebrecht (18141889). In: Münchner Historiker zwischen Politik und Wissenschaft. 150 Jahre Historisches Semi- 
Aber auch die Frage, wie singulär das Jubiläum im universitären Festkalender tatsächlich auftrat, bedarf der Abwägung. Für die Münchner Universität und ihr alljährliches, bis auf wenige Ausnahmen stets Ende Juni begangenes Stiftungsfest hat Katharina Weigand die Annahme erwogen, ob „immer und immer wieder, Jahr für Jahr, die Gründung der Ludwig-Maximilians-Universität im Jahre 1472 selbst oder zumindest Aspekte ihrer Entwicklung thematisiert worden“ sein könnten. ${ }^{9}$ Doch trotz des Anlasses der Universitätsbegründung trat das Stiftungsfest nicht als eine Art ,jährlich wiederkehrende Jubiläumsveranstaltung“ in Erscheinung. Nicht die Geschichte und Entwicklung der Institution, ihre Gegenwart oder Zukunft prägten für gewöhnlich die Stiftungsfeste an der Ludwig-Maximilians-Universität zwischen 1871 und 1918. Vielmehr dominierten in weitem Übermaße die von allen anderen akademischen Feiern, insbesondere den Rektoratsantritten, wohlbekannten Fachreden, deren Kernanliegen die Selbstdarstellung wechselnder Fächer vor der universitären und weiteren Öffentlichkeit war, mithin: der Alltag universitärer Redekultur. ${ }^{10}$ Doch ob der amtierende Rektor nun seiner fachlichen Herkunft oder der Geschichte seiner Alma Mater die Ehre erwies - die Verwandtschaft, die Nähe zwischen den alljährlich und den womöglich nur alle fünfzig oder hundert Jahre gehaltenen Universitätsreden liegt gleichwohl auf der Hand: Als „Ort“ der institutionellen Vergemeinschaftung, wozu selbstredend auch die fachliche Reflexion und Präsentation zählte, wurde die Ansprache an die versammelten Universitätsangehörigen als Nukleus der kommunikativen Selbstverständigung vor aller Öffentlichkeit begriffen. Die Auskunft über Imaginationen universitärer Vergangenheit(en), Gegenwart und Zukunft wurde dabei je nach Zuschnitt mehr oder weniger in den Mittelpunkt gerückt.

nar der Ludwigs-Maximilians-Universität. Hrsg. von Katharina Weigand. München 2010. S. 119136; zu seinem Nachfolger als Redner vgl. Graf, Friedrich Wilhelm: Ignaz von Döllinger (17991890). In: ebd. S. 57-77.

9 Weigand, Katharina: Münchner Rektorats- und Universitätsreden 1871-1918. In: Der rhetorische Auftritt. Redekultur an der Ludwig-Maximilians-Universität München. Rektorats- und Universitätsreden 1826-1968. Hrsg. von Claudius Stein. München 2016. S. 105-117, hier zum Stiftungsfest S. $111 \mathrm{f}$.

10 Auch die Möglichkeit, dass sich Rektoren, „die keine Historiker waren, einfach nicht zutrauten, niveauvoll genug die Geschichte der eignen Universität dazustellen“, verwirft Weigand, es handele sich bei dem Redenkorpus schlicht um eine „bunte thematische Mischung“ (Weigand, Rektorats- und Universitätsreden (wie Anm. 9), S. 112); weitere Beiträge zum hier behandelten Untersuchungszeitraum steuerten bei: Putz, Hannelore: Münchner Rektorats- und Universitätsreden 1826-1848. In: Stein, Auftritt (wie Anm. 9), S. 79-93; Körner, Hans-Michael: Münchner Rektorats- und Universitätsreden 1848-1871. In: Stein, Auftritt (wie Anm. 9), S. 95-104; nicht auf München beschränkt vgl. Haupt, Selma: Angetreten, um die Universität zu vertreten. Deutsche Rektoratsantrittsreden 1871-1918. In: Stein, Auftritt (wie Anm. 9), S. 49-59. 


\section{Jubiläen und ihre Ansprachen an der Münchner Universität im 19. Jahrhundert}

Der in München in besonderer Weise verdichtete Jubiläumskalender offerierte auffallend viele Gelegenheiten, ein Jubiläum zu begehen. Der Grund hierfür liegt im ungewöhnlichen, zweimaligen Umzug der Universität: Neben der üblicherweise den Anlass für ein Jubiläum bietenden Universitätsgründung - diese war 1472 in Ingolstadt erfolgt - bot sich der Münchner Universität zusätzlich die Möglichkeit, sowohl den 1800 vorgenommenen Wechsel vom Gründungsort nach Landshut sowie die 1826 erfolgte Translokation der Institution von Landshut nach München zu begehen. Denkbar waren demnach Feierlichkeiten, die sich auf die Jahre 1472, 1800 und 1826 bezogen - doch welche Jubiläen beging die LudwigMaximilians-Universität tatsächlich? Der erste Umzug der Universität von Ingolstadt nach Landshut im Jahr 1800 vermochte offenkundig nicht, den „Rang“ der Jubiläumswürdigkeit zu erlangen. Vermutlich vor allem deshalb, weil mit dem Weiterzug der Universität nur 26 Jahre später es an einer Gegenwart mangelte, die sich auf das historische Ereignis beziehen, aus diesem Sinn stiften konnte. Ein Jubiläum braucht nicht nur einen historischen Anlass, sondern auch eine jeweils gegenwärtige Initiative, die sich von der Feier des Jubiläums etwas verspricht.

Wenn auch das Jahr 1800 im Festkalender keine Beachtung fand, so wurde dem universitätshistorischen Ereignis des Umzuges durchaus Interesse entgegengebracht. Es bedurfte allerdings eines entsprechend motivierten Protagonisten. In der Rangfolge der Ämter lag es daher vor allem in der Hand des Rektors, ob sich die Wiederkehr einer geeigneten Begebenheit auch tatsächlich zur Festlichkeit auswuchs. Im Jahr 1900 jedoch mochte der amtierende Rektor, der Völkerrechtler Emanuel Ullmann, lieber über den „deutschen Seehandel und das Seekriegs- und Neutralitätsrecht" als über den Ortswechsel der Universität hundert Jahre zuvor sprechen. Nun handelte es sich zwar um eine der als seriell aufgefassten Reden zum Rektoratsantritt, mithin die historische Sinnstiftung nicht zwingend zu erwarten gewesen war. Gleiches aber traf auch drei Jahre zuvor zu. Im November 1897 war der als Rektor amtierende Historiker Karl Theodor von Heigel ebenfalls seinen eigenen, jedoch gänzlich anders gelagerten Interessen gefolgt und hatte sich in seiner Antrittsansprache just die „Verlegung der Ludwigs-Maximilians-Universität nach München“ zum Thema erkoren. Nicht nur diesen zweiten Umzug der Universität 1826, auch den ersten Ortswechsel von Ingolstadt nach Landshut 1800 thematisierte Heigel eingehend - drei Jahre „zu 
früh“. ${ }^{11}$ Ein Jubiläum ließ sich auf diese Weise jedenfalls nicht stiften, trotz einer Rede, die eines solchen würdig gewesen wäre. Überdies mag fraglich bleiben, ob die Universität sich dazu veranlasst gesehen hätte, wenn der fachlich ausgewiesene und interessierte Rektor sowie das denkbare Jubiläumsjahr zusammengetroffen wären. Nicht jede Gelegenheit wollte ergriffen werden, eine inflationäre Verbreitung von Jubiläen hätte dem seiner Natur nach seltenen - „singulären“ Ereignis entgegengestanden.

Auch das 1822 zumindest numerisch anstehende Jubiläum der 350 Jahre zurückliegenden Universitätsgründung konnte keine Wirkungskraft entfalten. Der wichtigste Grund dafür wird gewesen sein, dass sich die Universität inmitten von inhaltlichen und organisatorischen Reformprozessen befand, die sie seit der zweiten Hälfte des 18. Jahrhunderts „in ihrem inneren Bestand elementar“ veränderten. Vier Jahre nach dem „verpassten“ Jubiläum vollzog der Umzug in die Landeshauptstadt der bayerischen Monarchie, begriffen als „harter Schnitt“, auch äußerlich unübersehbar den institutionellen Wandel. ${ }^{12}$ Der mit einer Jubiläumsfeier verbundene Blick auf Herkunft und Entwicklung der Institution jedoch war vor allem der feierlichen Selbstbestätigung gewidmet, weniger der kritischen Reflexion.

Gleichwohl konnte der Rekurs auf die Gründung der Universität durchaus dazu dienen, nach krisenhaften Zeiten neuerlich Orientierung und Gewissheit zu finden. Zum Stiftungsfest im Juni 1849 sah sich der als Rektor amtierende Theologe Max Stadlbauer eben dazu gedrängt, nachdem er angesichts der „täglichen die Universität tief berührenden und in ihren Fundamenten erschütternden Vorkommnisse, und gegenüber den schweren Bedrängungen des akademischen Lebens und Wirkens“ zunächst versucht gewesen war, die „diesjährige StiftungsFeier gänzlich zu umgehen und einzustellen“. In „dieser Zeit, die keine Zeit der Gründung und der Stiftung, sondern der Zerstörung und Auflösung“ sei, wollte Stadlbauer den Erschütterungen der 1848er-Revolution, in deren in Bayern vergleichsweise moderatem Verlauf es auch zur Schließung der Münchner Universität gekommen war, mit einer Ansprache über „die Stiftung und älteste Verfassung der Universität Ingolstadt“ begegnen. Er hatte sich entschlossen, seinen

11 Ullmann, Emanuel: Der deutsche Seehandel und das Seekriegs- und Neutralitätsrecht. München 1900; Heigel, Karl Theodor: Die Verlegung der Ludwigs-Maximilians-Universität nach München. München 1897.

12 Vgl. Putz, Münchner Rektorats- und Universitätsreden (wie Anm. 10). S. 79; auch im mehrfach zitierten Band zu den Münchner Rektorats- und Universitätsreden, darauf verweist Putz ausdrücklich, wurden die in Landshut gehaltenen Reden nicht in einem eigenen Beitrag gewürdigt (in der digitalen Sammlung des Münchner Universitätsarchivs sind immerhin sechs Rektoratsantrittsreden sowie 24 sogenannte „Gelegenheitsreden“ aus dem Zeitraum vor 1826 verfügbar). 
„Blick zunächst von der Gegenwart und in das ,finstere Mittelalter zurück der Stiftung unserer hohen Schule zuzuwenden, um die Grundzüge der ursprünglichen und ältesten für die Universität geltenden, bei weitem nicht genugsam bekannten und gewürdigten Verfassungsbestimmungen mit den theils bereits errungenen, theils noch erwarteten Resultaten der neuesten Reformbestrebungen zu vergleichen" ${ }^{13}{ }^{13}$ Eine solche, dezidiert durch eine als krisenhaft empfundene Gegenwart zur universitätshistorischen Reflexion veranlasste Ansprache wäre mit den Usancen eines Jubiläums unweigerlich in Konflikt geraten. Zum „Glück“ stand für die Münchner Universität im Jahr 1849 trotz mehrerer möglicher Bezugsereignisse kein solches an.

Die Rahmenbedingungen, das kann für die Betrachtung von Hochschuljubiläen nicht genug betont werden, mussten passen, damit eine wie auch immer begründete „runde“ Anzahl von Jahren als Jubiläum in das universitäre Gedächtnis eingehen konnte. Als 1872, fünfzig Jahre nach dem nicht begangenen Jubiläum von 1822, die noch eindrücklichere Zahl von vierhundert Jahren seit der Universitätsgründung vergangen war, trat eben dieser Fall in München ein: Nach einigen Umwegen war auch die bayerische Monarchie Teil der „kleindeutschen Lösung “ der nationalen Frage geworden und die bereits erwähnten, entsprechend gestimmten Rektoratsreden der Jahre 1870 und 1871 hatten gleichsam den Boden für das 1872 anstehende Jubiläum bereitet. Nur vier Jahre darauf gelang es dem nächsten Anlass - fünfzig Jahre lag nun der für das Selbstverständnis der LudwigMaximilians-Universität eminent bedeutsame Umzug nach München zurück nicht, diese Hürde zu überspringen. Vielleicht war man des Feierns noch müde, vielleicht erschien die Zahl dann doch zu unbedeutend.

Im Jubiläumsjahr 1872 amtierte der bereits erwähnte katholische Theologe Ignaz von Döllinger als Rektor und übernahm die Ansprache anlässlich der 400jährigen Stiftungsfeier der Universität am 1. August. Welche Schwerpunkte setzte Döllinger, in welchem Verhältnis bewegte sich seine - einmalige, singuläre Ansprache zu den anderen Münchner Universitätsreden zu Beginn der 1870er Jahre? Wie alle anderen erwähnten Jubiläumsredner empfand auch Döllinger den Blick zurück in die Vergangenheit als zunächst gegebene Perspektive. Die Zeit sei dazu „angethan“, sich „aufzurichten an geschichtlichen Erinnerungen, auf dass wir im Spiegel der Geschichte um so heller die Gegenwart und die den Hochschulen in ihr gegebenen Zielpunkte und Aufgaben erkennen." ${ }^{\text {14 }}$

13 Stadlbaur, Max: Ueber die Stiftung und älteste Verfassung der Universität Ingolstadt. München 1849. S. $3 \mathrm{f}$.

14 Döllinger, Ignaz von: Festrede zur 400jährigen Stiftungsfeier. München 1872 (alle Zitate dort); zum Münchner Jubiläum von 1872 vgl. Müller, Winfried: Inszenierte Erinnerung an welche Traditionen? Universitätsjubiläen im 19. Jahrhundert. In: Die Berliner Universität im Kontext der 
Der Münchner Universität sei, schritt Döllinger weiter, das „Loos zugefallen“, als erste Universität im „neu geeinten Reich“ ihr Stiftungsandenken zu begehen. Das klang nun mehr nach einer Bürde als nach einem großen Glück, offenkundig der besonderen Rolle Bayerns im Reichsgründungsprozess geschuldet. Auch wenn ein vierhundert Jahre zurückliegendes Ereignis begangen wurde, blieb der zeitgenössische Kontext wirkmächtig: Bayern hatte immerhin noch 1866 an der Seite Österreichs gegen Preußen gekämpft, war dann aber gegen Frankreich auf Preußens Seite gewechselt. Auch die Münchner Rektoratsreden der beiden Vorjahre hatten mit der Herausforderung gekämpft, im Schatten dieser Konstellation Gemeinschaft und Sinn zu stiften. Gewunden nahm etwa der Historiker Wilhelm Giesebrecht im Dezember 1870 die durchaus heikle Entwicklung auf und warb um Anerkennung für die Leistungen des bayerischen Heeres, um möglichst rasch wieder sichereres Terrain zu suchen: Giesebrecht hoffte in seiner Rede, „spätere Geschlechter“ würden „einst von der Universität in Bayerns Hauptstadt rühmen, dass sie in der großen Zeit nationaler Wiedergeburt Geister geweckt, deren Wirken nicht Bayern allein, sondern dem ganzen Deutschland Gewinn gebracht" habe. ${ }^{15}$

Die Alma Mater als sicherer Hort - und als Ebenbild der Nation, diesen Weg beschritt auch der Theologe Döllinger gut eineinhalb Jahre darauf, war die Münchner Universität doch „eine Körperschaft, zusammengeschlossen aus allen Gauen Deutschlands, und fort und fort sich ergänzend von Nord und Süd, Ost und West." Als nicht nur universitätsgeschichtlich vorbildhaft zeichnete Döllinger die „starke corporative Verfassung“ der mittelalterlichen Universitäten, gleich einer „wohl befestigten Burg“. Für die deutschen Universitäten habe ihr Aufstieg mit dem 18. Jahrhundert begonnen, bis ,endlich in den Gründungen von Berlin und Bonn das Höchste, was das 19. Jahrhundert in Deutschland auf diesem Gebiete erwarten konnte, geleistet wurde, das ist bekannt und oft besprochen." Weitere Worte $\mathrm{zu}$ den preußischen Leistungen verlor Döllinger nicht, auch der Name Humboldt blieb ungenannt, wie auch sonst in den gesichteten Rektorats- und Universitätsreden in München. ${ }^{16}$

Der sichere Boden des Jahres 1872 (,Wir dagegen blicken jetzt festen Muthes und ruhigen Vertrauens in die Zukunft, denn die Sehnsucht nach dem Reiche,

deutschen Universitätslandschaft nach 1800, um 1860 und um 1910. Hrsg. von Rüdiger vom Bruch. Unter Mitarbeit von Elisabeth Müller-Luckner. München 2010 (Schriften des Historischen Kollegs, Kolloquien 76). S. 73-92, hier S. 80-84, $89 \mathrm{f}$.

15 Giesebrecht, Einfluß (wie Anm. 8), S. 27.

16 Vgl. Paletschek, Sylvia: Verbreitete sich ein ,Humboldtsches Modell‘ an den deutschen Universitäten im 19. Jahrhundert? In: Humboldt international. Der Export des deutschen Universitätsmodells im 19. und 20. Jahrhundert. Hrsg. von Rainer Christoph Schwinges. Basel 2001. S. $75-$ 104; sowie Langewiesche, Humboldt als Leitbild? (wie Anm. 7). 
die allen Deutschen tief in's Herz gegraben, ist erfüllt.") erlaubte Döllinger schließlich einen Appell zur kritischen Selbstsicht. Sollte man nicht, schloss er seine Ansprache, auch ,einen vergleichenden Blick auf die Hochschulen stammverwandter Völker werfen und in diesem Spiegel das, was uns mangelt beschauen? Wir finden im ganzen Westen [...] das Collegiensystem. Die höheren Schulen sind nicht blos Lehr-, sondern auch Erziehungs-Institute.“ Vor allem England und die Vereinigten Staaten könnten als Vorbild dienen. ${ }^{17}$ Der Idee war kein Nachhall im deutschen Bildungswesen beschieden. Immerhin inspirierte Döllingers singuläre Jubiläumsansprache den nach dem Universitätskalender folgenden Redner. Auch der im Herbst 1872 das Rektorat der Münchner Universität antretende Jurist Wilhelm Planck referierte über die Geschichte der Universität als Institution der Bildung und Erziehung. Eine eigentliche Serie sollte daraus allerdings nicht werden, denn die Mehrzahl der in den folgenden Jahren antretenden Rektoren bevorzugte es, ihr eigenes Fachgebiet vorzustellen.

Die eminente, ja konstitutive Bedeutung der jeweiligen Gegenwart für die Ausprägungen einer universitären Jubiläumskultur verdeutlichen auch die nächsten beiden „runden“ Jahrestage der Ludwig-Maximilians-Universität in den Jahren 1922 und 1926. Vier Jahre nach dem Ende des Ersten Weltkrieges fiel das 450-jährige Gründungsjubiläum angesichts knappster Mittel ausgesprochen bescheiden aus, zudem war das Jubiläum in unmissverständlicher Opposition zu den Zeitläufen begangen worden: Die Festveranstaltung sparte die schwarz-rotgoldene Nationalflagge der Weimarer Republik aus, und der abendliche studentische Festkommers ließ sich von der eintreffenden Nachricht der Ermordung des Reichaußenministers Walther Rathenau nicht stören. ${ }^{18}$ Die Festansprache widmete der Geograph Erich von Drygalski, durchaus einem Jubiläum angemessen, den „Zeitfragen der Universität“. Ähnlich wie bei zahlreichen Ansprachen zum Beginn der 1920er Jahre führte auch Drygalskis Blick zurück in die für viele Akademiker weiterhin sinnstiftende Zeit des Kaiserreichs. Doch nicht ausschließlich Jahrzehnte oder Jahrhunderte zurückliegende Stationen der universitären Fest- und Redehistorie versprachen, Orientierung zu geben. Ausdrücklich

17 Döllingers Appell ist auch als Wendung gegen eine zuvor erfolgte Entwicklung zu verstehen: Hannelore Putz hat für die Universitätsreden des Zeitraums zwischen 1826 und 1848 festgestellt, dass sich in diesen Jahren das Selbstverständnis der Ludwig-Maximilians-Universität gewandelt habe, man begriff sich zunehmend als Institution der akademischen Lehre, weniger als Erziehungsanstalt, vgl. Putz: Münchner Rektorats- und Universitätsreden (wie Anm. 10), S. 93.

18 Vgl. Schreiber, Maximilian: Die Ludwig-Maximilians-Universität und ihre Jubiläumsfeiern in der ersten Hälfte des 20. Jahrhunderts. In: Die Universität München im Dritten Reich. Aufsätze Teil I. Hrsg. von Elisabeth Kraus. München 2006 (Beiträge zur Geschichte der Ludwig-MaximiliansUniversität München 1). S. 479-504, hier S. 481-486. 
gedachte Drygalski der 1913 an der Universität begangenen ,Jahrhundertfeier der Befreiungskriege“ und der damaligen Ansprache Karl Theodor von Heigels. ${ }^{19}$ Die Erinnerung an ein noch in vermeintlich ungebrochener Einigkeit - der Lehrkörper dürfte trotz der Kriegsverluste wesentlich noch jenem neun Jahre zuvor entsprechen - gefeiertes Jubiläum sollte das Fest des Jahres 1922 stützen. Die „deutsche Erhebung vor hundert Jahren und heute“, das Sehnen nach einer erfolgversprechenden historischen Analogie zählte zum festen Inventar der Beschwörung nationalen Wiederaufstiegs. ${ }^{20}$

Auch 1926, als in den „guten“ Jahren der Weimarer Republik die 100-JahrFeier des Umzugs der Universität in ein zünftiges Jubiläum mündete, sind die Rahmenbedingungen nicht zu unterschätzen. Der amtierende Rektor, der Romanist Karl Vossler, stand der Weimarer Republik zustimmend gegenüber und ergriff die Chance einer umfangreichen Presseberichterstattung, gar einer Rundfunkübertragung des Festaktes, um die Münchner Universität mit dem Jubiläumsakt in der Weimarer Republik ankommen zu lassen. ${ }^{21}$ Überdies beließ es Vossler nicht bei der weihevollen Repräsentation der Universität in der städtischen und weiteren Öffentlichkeit. Im Anlass für das Jubiläum, der Translokation der Universität nach München, erkannte Vossler vielmehr die Chance zur gegenwarts- und zukunftsorientierten Sinnstiftung. Man feiere, schrieb er seinen keineswegs einflusslosen Gegnern mit seiner Festansprache ins Stammbuch, die „Überwindung des geistigen Provinzialismus“ und sende deshalb „den Gruß der geistigen Freiheit an die Mitarbeiter in der Fremde, an die unprovinzialen Gehirne und Herzen unter den Forschern aller Völker und Rassen. “22 Nachdem die Jubiläumsfeier vier Jahre zuvor „Weimar“ als Staat wie als Idee gänzlich ignoriert hatte, feierte die Münchner Universität nun unter deutlich verändertem Banner.

19 Drygalski, Erich: Zeitfragen der Universität. Rede zum 450jährigen Jubiläum der LudwigMaximilians-Universität München. München 1922. S. 1; vgl. auch Maurer, Trude: Engagement, Distanz und Selbstbehauptung. Die Feier der patriotischen Jubiläen 1913 an den deutschen Universitäten. In: Jahrbuch für Universitätsgeschichte 14 (2011). S. 149-164.

20 Vgl. Müller, Karl Alexander von: Die deutsche Erhebung vor hundert Jahren und heute. In: Süddeutsche Monatshefte 21 (1923/24). S. 131-145; Wenzel, Kay: Befreiung oder Freiheit? Zur politischen Ausdeutung der deutschen Kriege gegen Napoleon von 1913 bis 1923. In: Griff nach der Deutungsmacht. Zur Geschichte der Geschichtspolitik in Deutschland. Hrsg. von Heinrich August Winkler. Göttingen 2004. S. 67-89.

21 Vgl. Schreiber, Jubiläumsfeiern (wie Anm. 18), S. 489-494.

22 Vossler, Karl: Ansprache beim Festakt der Jahrhundertfeier am 27. November 1926. In: ders.: Politik und Geistesleben. Rede zur Reichsgründungsfeier 1927 und drei weitere Ansprachen. München 1927. S. $16 \mathrm{f}$. 


\section{Weder Blaupause noch Kontrastfolie - Jubiläumsansprachen an der Technischen Hochschule München}

Neben dem diachronen Vergleich verschiedener Münchner Universitätsjubiläen des 19. Jahrhunderts kann möglicherweise auch ein synchroner Blick auf eine weitere Institution helfen, sich den Fragen dieses Beitrages zu nähern. Wie verhielt es sich bei einer weitaus weniger traditionsversicherten Institution wie der Technischen Hochschule in München? Wie lassen sich ihre Ansprachen einordnen, zumal angesichts des unvermeidlichen Blickes auf den sehr viel älteren und (noch) unvergleichlich größeren Kontrahenten? In der Tat ergab sich eine gewisse Parallelentwicklung zur „großen“ Ludwig-Maximilians-Universität: Nur ein Jahr nach deren Umzug von Landshut nach München 1826, die wie erwähnt zu einer Reihe von bildungspolitischen Richtungsentscheidungen zählte, kam es 1827 in München zur Gründung der „Polytechnischen Centralschule“, einer der im 19. Jahrhundert zunehmend aufkommenden Ingenieurschulen. ${ }^{23}$ Die ab 1877 schließlich als Technische Hochschule bezeichnete Institution ging aus einer 1868 neu gegründeten polytechnischen Schule in München hervor. Es handelte sich bei der Technischen Hochschule demnach um eine ausgesprochen junge Einrichtung, als 1872 die Ludwig-Maximilians-Universität ihre 400-Jahr-Feier beging. ${ }^{24}$

Doch immerhin jährte sich 1877, eben im Jahr der Umbenennung zur Technischen Hochschule, die Gründung der früheren polytechnischen Centralschule München zum fünfzigsten Male. Der als Rektor der Technischen Hochschule amtierende Historiker August von Kluckhohn ließ sich eine solche, noch ausgesprochen seltene Chance zur geschichtlichen Einordnung nicht entgehen. Ausdrücklich als Jubiläum benannt wurde der Anlass nicht, auch handelte es sich um Kluckhohns Ansprache zum Antritt des Rektorats. Doch schon im Titel der Rede „Über das technische Unterrichtswesen in Bayern bis zur Gründung der poly-

23 Einführend vgl. König, Wolfgang: Zwischen Verwaltungsstaat und Industriegesellschaft. Die Gründung höherer technischer Bildungsstätten in Deutschland in den ersten Jahrzehnten des 19. Jahrhunderts. In: Berichte zur Wissenschaftsgeschichte 21 (1998). S. 115-122; zur Entwicklung in Bayern vgl. Fisch, Stefan: „Polytechnische Schulen“ im 19. Jahrhundert. Der bayerische Weg von praxisorientierter Handwerksförderung zu wissenschaftlicher Hochschulbildung. In: Die Technische Universität München. Annäherungen an ihre Geschichte. Hrsg. von Ulrich Wengenroth. München 1993. S. 1-38.

24 Zur weiteren Geschichte der Münchner Technischen Hochschule respektive Universität vgl. Dienel, Hans-Liudger u. Helmut Hilz (Hrsg.): Bayerns Weg in das technische Zeitalter - 125 Jahre Technische Universität München 1868-1993. München 1993. 
technischen Centralschule in München (1827)“ - verwies das eigens vermerkte Gründungsjahr darauf, dass es dem Redner auf eine historisch orientierte Sinnstiftung ankam. ${ }^{25}$ Er wolle die Aufmerksamkeit seiner Zuhörer auf „die Geschichte jener Studien lenken, für deren Pflege unsere Hochschule vornehmlich gegründet worden ist.“

Zunächst war Kluckhohn bemüht, den zeitlichen Rahmen seiner Betrachtungen zu weiten und setzte mit der Begründung praktisch orientierter Realschulen im 18. Jahrhundert ein, bei deren Einrichtung es allerdings in Bayern einige Mängel gegeben habe. Die Notwendigkeit eines technischen Unterrichtswesens traf in Kluckhohns Historie auf einen klerikal geprägten Bildungssektor, der je nach Einsatz des jeweiligen Monarchen seine Beharrungskraft entfalten konnte. Kluckhohn benannte die Gründe, die für eine praktisch ausgerichtete, aber zugleich höhere Ausbildung sprachen, auf eine weiter ausgreifende Sinnstiftung verzichtete er jedoch. Dass 1827 eine Gewerbeschule entstand, war in Kluckhohns Narrativ gleichwohl folgerichtig. Vergebens habe der erste Direktor der Schule, der einflussreiche Techniker und Unternehmer Joseph von Utzschneider, auf eine „Verbesserung der zuerst nur provisorisch getroffenen Einrichtungen“ gehofft, doch was Utzschneider „ersehnte, ist seit 9 Jahren, Dank der weisen Regierung Sr. Majestät unseres hochgesinnten Königs, [...] herrlich in Erfüllung gegangen. Wir haben eine Hochschule aller technischen Disciplinen“, die sich trotz ihrer „Jugend“ nicht „unwerth dünkt, von den altehrwürdigen Universitäten als die jüngere Schwester anerkannt zu sein“.

Im Willen, den Rang seiner Institution möglichst hoch anzusiedeln, kam Kluckhohn schließlich im letzten Satz seiner Ansprache den traditionellen Formen der Universitätsrede so nahe, wie er es sich für seine Hochschule als Wissenschaftseinrichtung zuvor ersehnt hatte: „Aber wollen Sie nicht vergessen, meine jungen Freunde, dass mit der höheren und freieren Stellung auch die Selbstverantwortung des Mannes wächst. Sie sind Studierende einer Hochschule: bleiben Sie immer dessen würdig!“ Als angehender Rektor bedachte er die Studierenden mit einer „Ermahnungs-Rede“, welche mehr als vierhundert Jahre zuvor bereits 1472 im Stiftungsbrief der damals Ingolstädter, nun Münchner, Universität dem Rektor vorgeschrieben worden war und seitdem bis weit in das 19. Jahrhundert zu den festen Bestandteilen aller Ansprachen zum Rektoratsantritt gezählt hatte.

25 Kluckhohn, August: Über das technische Unterrichtswesen in Bayern bis zur Gründung der polytechnischen Centralschule in München (1827). Antrittsrede gehalten am 22. Dezember 1877 von dem derzeitigen Director der technischen Hochschule. München 1878 (alle Zitate dort). 
Bereits im Juli 1879 setzte Kluckhohn seine institutionsgeschichtliche Reflexion vor der versammelten Gemeinschaft der Lehrenden und Studierenden fort. ${ }^{26}$ Vor zehn Jahren habe man beschlossen, jedes Jahr zu dieser Zeit in „feierlicher Versammlung“ die Resultate der von den Abteilungen „veranlassten Preisbewerbung“ zu verkünden. Doch sei es beim Vorhaben geblieben, der „unfertige Zustand der Aula“ habe die Begründung dieser Sitte verhindert, an die „Stelle des beabsichtigten Festactes trat die Proclamation der Preisträger durch Anschlag am schwarzen Brett.“ Auf diese wenig festliche Weise, das war Kluckhohn vollkommen klar, ließ sich weder eine Tradition noch der Anlass für ein künftiges Jubiläum stiften. Seine Rede sollte deshalb einen neuen Versuch starten, eine solche Feier in den Jahreskalender der Technischen Hochschule aufzunehmen. Durchaus passend sprach Kluckhohn deshalb über die „Gründung und bisherige Entwicklung“ seiner Hochschule, nachdem sein Vortrag über ihre Vorgeschichte, der Hinweis erschien ihm offenkundig unverzichtbar, sich der „Aufmerksamkeit einer hochansehnlichen Zuhörerschaft“ erfreut hatte. Es lag in der Natur der Sache, dass Kluckhohns Vortrag nur wenig Historie und sehr viel Gegenwart bot, letztere zudem teils von eher betrüblicher Natur war, da die anhaltende Gründerkrise die Attraktivität technischer Ausbildungsgänge erheblich gemindert hatte.

Der feste Willen, trotz einer noch kaum vorhandenen Institutionsgeschichte, Jubiläen zu begehen - mithin Geschichtlichkeit zumindest zu behaupten -, kennzeichnete die Münchner Technische Hochschule von Beginn an. ${ }^{27}$ Früh orientierte sich diese an der für die altehrwürdigen Universitäten als „permanente Erfindung einer Tradition“ bezeichneten unablässigen Beschwörung von institutioneller Vergangenheit, Gegenwart und Zukunft. ${ }^{28}$ Anlässlich des Semesterbeginns im Herbst 1893 wurde die lediglich 25 Jahre zurückliegende Gründung der polytechnischen Hochschule begangen. Die ausdrücklich als „Festrede“ bezeichnete Ansprache übernahm, ganz wie bei der Ludwig-Maximilians-Universi-

26 Kluckhohn, August: Ueber die Gründung und bisherige Entwicklung der k. technischen Hochschule zu München. Rede gehalten von dem derzeitigen Director am 26. Juli 1879. München 1879 (alle Zitate dort); zum zeitgenössischen „Publikationswesen zu Polytechnika und Technischen Hochschulen“, in dem München keine erwähnenswerte Rolle spielte, vgl. Guhl, Anton F.: Technik als blinder Fleck in der Universitätshistoriographie? Die Debatte um die Gründung von Polytechnika Anfang des 19. Jahrhunderts und ihre Ausblendung durch die Universitätsgeschichte. In: Hochschulen im öffentlichen Raum. Historiographische und systematische Perspektiven auf ein Beziehungsgeflecht. Hrsg. von Martin Göllnitz u. Kim Krämer. Göttingen 2020 (Beiträge zur Geschichte der Universität Mainz Neue Folge 17). S. 81-99.

27 Siehe den Beitrag von Anton F. Guhl in diesem Band.

28 Vgl. Paletschek, Sylvia: Die permanente Erfindung einer Tradition. Die Universität Tübingen im Kaiserreich und in der Weimarer Republik. Stuttgart 2001 (Contubernium. Tübinger Beiträge zur Universitäts- und Wissenschaftsgeschichte 53). 
tät anlässlich der gut zwanzig Jahre zuvor abgehaltenen 400-Jahr-Feier, der amtierende Rektor, der Mineraloge Karl von Haushofer. ${ }^{29}$ Doch blieb der singuläre Anlass hier eingebettet in die serielle Reihe der Reden zur Eröffnung des Studienjahres, überdies konnte Haushofer bereits in seinen einleitenden Worten die offenkundig gehegten Zweifel, ob es bereits angängig sei, ein Jubiläum zu begehen, nicht verbergen: Zwar seien die vergangenen 25 Jahre „für das Leben unserer Hochschule vielleicht von grösserer Wichtigkeit [...] als es die nächstkommenden drei Vierteljahrhunderte sein werden“, da es zunächst zu beweisen galt, dass man der „übertragenen Mission“ gerecht zu werden vermochte. Trotzdem habe das Kollegium beschlossen, von einer besonderen Feier abzusehen. Bescheiden klang die von Haushofer ausgegebene Begründung allerdings nicht: Es seien die „Grundlagen“, auf denen die Hochschule „aufgebaut und eingerichtet“ wurde, doch „für alle Zeiten bleibende“. Diese sicherten „unserer Anstalt eine Dauer [...], für deren Bemessung ein Vierteljahrhundert einen zu kleinen und kleinlichen Massstab“ abgebe.

Mithin, nur so war der Rektor zu verstehen, entsprach die tatsächlich als Institution bereits gewirkte Zeit nicht dem ihr zugedachten und zugesicherten Zeithorizont - man war offenbar fest davon überzeugt, dass sich diese Lücke mit dem unvermeidlichen Fortgang der Zeit schließen und der Hochschule ein auch nominell würdiges Jubiläum ermöglichen würde. Auch Haushofer hielt sich nicht mit der Jugend seiner Institution auf und erweiterte seinen „Bericht über die Thätigkeit der Hochschule im abgelaufenen Studienjahre“ um einen „Rückblick auf die verflossenen fünfundzwanzig Jahre“ der Hochschule, ihrer Entwicklung und Leistungen, für die ihm der übliche Geschäftsbericht eines Studienjahres offenbar $\mathrm{zu}$ gering erschien. Folgerichtig setzte Haushofers Ansprache ausdrücklich mit der bereits von Kluckhohn - auf welchen Haushofer direkt verwies referierten, auch nach seinen Worten wenig vorbildhaften Vor-Geschichte technischer Ausbildung in München ein. Nicht die weltabgewandte Universität, sondern die fehlgeleitete, da an den Zwecken einer Gewerbeschule orientierte Ausrichtung der früheren polytechnischen Central-Schule diente als Kontrastfolie. Diese angesichts des erhobenen Anspruchs auf Wissenschaftlichkeit unbedingt zu bestreitende Herkunft wurde von Haushofer spöttisch karikiert:

Der Hochschule [...] kann unmöglich die Aufgabe zugedacht werden, manuell geschulte Techniker auszubilden und der Gedanke, den studierenden Maschineningenieur täglich einige Stunden an den Schraubstock und die Drehbank zu stellen, hat ungefähr den gleichen

29 Haushofer, Karl von: Rückblicke auf die Entwicklung der Königlichen bayerischen technischen Hochschule in den ersten 25 Jahren ihres Bestehens. Festrede gehalten zur Eröffnungs-Feier des Studienjahres am 18. November 1893. München 1894 (alle Zitate dort). 
Wert, wie etwa die Idee hätte, den studierenden Juristen täglich ein paar Stunden Schwurgerichts-Zeugenvernehmungen protokollieren zu lassen.

Einen vollkommen anderen Tonfall schlug Haushofer in der Beschreibung des Verhältnisses zur Ludwig-Maximilians-Universität an. Beide Hochschulen „blühen und gedeihen nebeneinander“, es habe sich eine „innige Wechselwirkung herangebildet“. Auf Augenhöhe mit der Universität wollte sich die Technische Hochschule beschreiben, die unbestreitbare Rangfolge wurde lyrisch verbrämt: „Sind auch in dem edlen Wettkampf Wind und Sonne hie und da nicht ganz gleich verteilt, so ist doch zu gewärtigen, dass seine Ergebnisse der Allgemeinheit, der Förderung der Wissenschaft und dem Landeswohle zu statten kommen." Den offenkundig weitaus weniger prosaischen Alltag konnte Haushofer in seiner Festrede nicht gänzlich übergehen. Wenig festlich etwa fiel seine Verteidigung der landwirtschaftlichen Abteilung der Hochschule aus. Rasch kehrte Haushofer zur Betonung der Wissenschaftlichkeit der an der Technische Hochschule vertretenen Fächer zurück und hob die Bedeutung des elektrischen Stroms für die Physik als Fach wie für die Technische Hochschule als Lehrgegenstand hervor, um schließlich - doch ganz der Form des Geschäftsberichtes erliegend - in großer Ausführlichkeit die Anzahl der Lehrenden, die im Gegensatz zur Universität nunmehr stetig zunehmende Studierendenfrequenz, ihr Verhältnis zur wirtschaftliche Entwicklung einzelner Industriezweige und zu guter Letzt auch die Entwicklung der Stipendien zu referieren.

Mit keiner Zeile suchte die Festrede Haushofers, den Kontrast zur Jubiläumskultur der Universität zu zeichnen. Als Blaupausen indes können die anlässlich von Universitätsjubiläen gehaltenen Ansprachen zugleich ebenfalls nicht begriffen werden: Vergangenheit, Gegenwart und Zukunft befanden sich hier in einem gänzlich anderen Verhältnis. Geschichtlichkeit, die den Universitäten wie ein schwerer Mühlstein um den Hals hing, kannte die Festrede Haushofers nur als in die Zukunft gerichtete Projektion: Wenn man weiterhin „das Höchste“ leiste, dann ,werden auch unsere Namen dereinst aus dem Staub der Archive zu neuem Leben auftauchen, dann wird man vielleicht auch von uns erzählen, dass wir an der Entwicklung und an der Ehre unserer Hochschule gebaut haben, auf dass sie fortdauere über die Jahrhunderte." Damit schloss Haushofer den - nun doch ebenso bezeichneten - „Festakt“ und bat die „hohen Festgäste“, zur „Enthüllung der Büste Gottfried von Neureuthers in das Treppenhaus folgen zu wollen." Der Architekt Neureuther hatte seit 1868 als einer der ersten Hochschullehrer am Polytechnikum gelehrt und nicht zuletzt das neue Hauptgebäude der jetzigen Technischen Hochschule gebaut. 


\section{Ausblick}

Die mit aller Macht, gegen den eigenen Zweifel betriebenen Bestrebungen der Technischen Hochschule, der eigenen Institution sobald als möglich die Jubiläumswürdigkeit zusprechen zu können, verweist auf die enorme Bedeutung von Jubiläen für die Sinnstiftung und Sinntradierung auch, aber selbstredend nicht allein von Hochschulen. Die feierliche Rede als eine der wichtigsten Formen akademischer Vergemeinschaftung bot dabei - im Gegensatz etwa zu Festschriften oder Festumzügen - die Chance, auf dem keineswegs eindeutigen Feld möglicher oder denkbarer, gewünschter oder unerwünschter Jubiläen einen gewissen Spielraum zu erlangen. Zwischen universitären Wünschen und außeruniversitären Zwängen, zwischen passenden Gelegenheiten, willigen oder unwilligen Protagonisten konnte eine Rede in vielfacher Weise ausgestaltet sein, sich am Geländer der Gewohnheit, der Serialität festhalten oder die Singularität eines „Jahrhundertjubiläums“ gebührend herausheben. Selbst den gemeinhin zu vermeidenden Mittelweg schloss die universitäre Rede keineswegs aus: Die Reflexion über Herkunft und Entwicklung, über Aufgabe und Zukunft der Institution vermochte die alljährliche Universitätsrede mit der eines Jubiläumsjahres zu verbinden, mithin Singularität und Serialität miteinander zu versöhnen.

Der Technischen Hochschule München allerdings blieb auch ihr nächstes, durchaus gerechtfertigtes Jubiläum versagt: Im November 1918 blieb die fünfzig Jahre zurückliegende Gründung der Hochschule ungefeiert. Auch die LudwigMaximilians-Universität konnte ihre Jubiläumskultur nicht ungehindert fortführen, die wechselhaften äußeren Bedingungen im München der 1920er Jahre, aber auch institutionelle Krisen setzten sowohl zu den gegeben Anlässen wie auch in allgemeinerer Weise die universitäre Redekultur unter Druck. Bereits im Weltkrieg war eine Vielzahl von Akademikern in zuvor ungekannter Weise zu Rednern in der politischen Öffentlichkeit avanciert, doch auch innerhalb der akademischen Zirkel verschärfte der Krieg die Verkehrsformen, bescherte der Redekultur eine nachdrückliche Politisierung.

Die universitäre Redekultur hatte sich ungewohnter Konkurrenz zu erwehren, zusätzlich verstärkt durch neue Medien wie dem Rundfunk, der den rasch aufkommenden modernisierungskritischen Zweifeln mit belehrenden Vorträgen ans breite Volk begegnete. Ohne Rückwirkung auf die Universitäten blieb die Ausweitung des medialen Feldes nicht, doch konnten diese von einer interessierten, politisierten und zunehmend medialisierten Gesellschaft auch profitieren, öffentliche Vorträge und politische „Erziehungsangebote“ von Professoren lockten neue Publika in die Hörsäle. Eine vitalisierte öffentliche Redekultur wirkte auf die Universität, bot ihr Entfaltungsmöglichkeiten und setzte sie zugleich unter den 
Druck konkurrierender Deutungsangebote. Die vielfach durch äußere Ereignisse unterbundene Serialität von Rektorats- und Stiftungsfestreden ließ diese, wenn sie denn stattfanden, singulären Gehalt erlangen, zudem suchten Redner nun auch zum profanen Anlass den grundsätzlichen Gestus. Die verdichtete, von Konkurrenzen und Konjunkturen geprägten Gedenkkultur der Weimarer Republik - die nur vier Jahre auseinander liegenden Jubiläen der Ludwig-MaximiliansUniversität von 1922 und 1926 offenbarten den breit gespannten Möglichkeitshorizont - ließen die Hochschulen in der Ausgestaltung ihrer Jubiläen in besonderer Weise zu „Geschichtemachenden“ werden. 